\title{
Operational and real-time LHD dispatch at Rio Tinto's Argyle Diamond Mine
}

\author{
SN Donaldson Polymathian Pty Ltd, Australia \\ W Harney Rio Tinto, Australia
}

T Cox Polymathian Pty Ltd, Australia

\begin{abstract}
This paper describes the configuration and implementation of both real-time and operational modules of an equipment dispatch tool to Rio Tinto's Argyle Diamonds. The tool, known as ORB, uses mathematical programming to optimise both operational equipment extraction node assignment, as well as realtime draw point dispatch. It is the first known optimisation-based, autonomous, real-time dispatch tool custom built for underground block cave mining operations. With ORB, Argyle Diamonds benefit from continuously and autonomously co-ordinating the optimal extraction node assignment and dispatch of LHDs in order to achieve a range of objectives while adhering to draw compliance and geotechnical constraints. The paper starts by outlining the pre-existing business planning process, detailing the simple logic that produced the daily draw order and equipment timeline. Next, the motivations for an optimisation-based decision-support tool are presented, drawing comparison to the limitations in existing planning processes. Following this, the optimisation tool developed is outlined, including the data it uses and the objectives it optimises. Finally, a case study of the implementation of the tool to Rio Tinto's Argyle Diamonds is presented, detailing the benefits it has brought alongside the unique constraints of this heavily restricted block caving environment.
\end{abstract}

\section{Introduction}

\subsection{Block cave extraction}

Load-haul-dump (LHD) machines extract material from a block cave by bogging from a draw point in an extraction drive, transiting a pre-defined tramming route, before dumping into a tip point (Hustrulid 2001). The LHDs cycle between draw points and tip points across a day, with the number of tonnes to be bogged from each draw point at any given time being directed by the draw strategy. LHD fleet management consists of dispatching, routing and scheduling (Paravarzar et al. 2018). The combination of LHD allocations to draw points determined by the dispatch decisions (called a 'bogging pattern') can be impacted by a range of factors including:

- Layout of the cave

- Potential congestion along tramming routes

- Secondary break activities

- Drive rehabilitation

- Materials handling system (MHS) throughput capacity

- Impact of wet material through the MHS

- Convergence of extraction drives and draw point access

- Stockpiles 
- Geotechnical constraints

- Strategic objectives including target grade profile

\section{$1.2 \quad$ Limitations of existing dispatch methods}

\subsubsection{Draw strategy}

Existing dispatch methods are typically based around daily tonnes targets for each draw point. Priorities are assigned at the start of the shift, and a 'draw call' issued to each LHD operator for the extraction drive they will start the day in. This creates a fixed daily planning cycle that resets at the start of the day shift, to which compliance is measured.

The ability to adapt and update a plan within the shift is limited by the constraints of those daily targets. As unexpected events occur throughout the day, the production supervisor is required to make dynamic dispatch decisions to attempt to meet daily compliance targets. These decisions are complex and need to consider a wide range of variables. The quality of these decisions is influenced by the experience of the production supervisor, the data available to them, and the time they have available to consider possible options.

This process can be especially limiting toward the end of the night shift when there may be limited tonnes remaining in the cave. LHDs can end up doing 'tidy up' work at the end of the night shift, as production supervisors adapt the plan to try and hit explicit daily targets over this artificially constrained planning horizon.

\subsubsection{Extraction drive allocation}

The first component of LHD dispatch is the decision of which extraction drives to allocate LHDs to across the day, and their corresponding tramming routes. This can be referred to as the equipment 'daily timeline'. Depending on the age and strategic objectives of a cave, this may be an incredibly complex or a trivial process.

Factors that contribute to the complexity of this process are:

- The frequency at which LHDs must change extraction drive:

o This is impacted by the number of LHDs vs number of available extraction drives, as well as general tonnage availability in each extraction drive.

- Requirement for material blend

o If tonnes must be blended into the materials handling system to either prevent issues from wetness, or meet a target ore blend, then it is important that this is considered in the extraction node allocation.

- Convergence

- When areas of the cave become inaccessible or have restricted access, tramming route compatibility between LHDs can be complex.

o For example, an extraction drive may have been plugged in the centre of the drive due to convergence. This results in some draw points not having a direct tramming route to a tip point. In this scenario, it is likely required to either utilise a stockpile (resulting in rehandling) or use another extraction drive as a tramming route (resulting in potential conflicts with another LHD).

- Various LHD models

- Some LHDs may be more efficient than others (larger buckets / faster) or have restrictions on what areas of the cave they can access based on their size. 
The process of creating and updating the 'daily timeline' can be a challenging, manual and time-consuming task. The quality of the plan is based on the experience of the engineer creating it and their available time. It should be completed every day to reflect the dynamic cave state and historical performance, however when it is particularly difficult to update or appropriate staff do not have available time, the timeline may not get updated for a longer period. This can result in updates only occurring when major changes to the cave occur, which means smaller limitations are tolerated to avoid the burden of creating a new timeline. In execution, this can result in a production supervisor defending the plan even when the assumptions built into it, such as the number of operating LHDs, have significantly changed.

\subsubsection{Draw point dispatch}

Given an allocation of LHDs to extraction drives, it is then required to dispatch the LHDs to individual draw points. When a daily draw order is in use, this is typically done by following a fixed order and tonnage for each draw point defined at the start of the shift. This order may be as simple as 'start from the footwall and progress to the hanging wall'. This process results in each LHD being treated individually and removes any potential to treat the LHD fleet as a team. This misses significant opportunities for optimisation and automation which can increase throughput, increase productivity, improve compliance, and achieve consistent target blend ratios.

\section{ORB: An optimisation-based decision support tool for extraction node assignment and LHD dispatch}

ORB is an optimisation-based decision support tool, which has been developed to solve multiple planning problems from real-time dispatch through to strategic mine planning in a range of hard rock underground mines. This includes solutions for both the extraction node assignment and LHD dispatch problem in a block cave, which are described below.

\subsection{Block cave draw strategy}

Core to the planning of any equipment dispatch decisions in a block cave is the draw point draw strategy which governs allowable tonnes to be bogged over time. A review of production scheduling methods in block caving (Khodayari \& Pourrahimian 2015) show that many approaches have been developed for setting long and medium term production targets. These targets are core input data to operational and real-time planning problems in ORB and encapsulate both geotechnical and planning constraints, as well as the strategic objectives for a cave.

ORB can be used to effectively map these long and medium term targets to dispatchable units of works. ORB allows draw point targets to be set over any explicitly defined time period. For example, monthly targets can be used instead of the traditional daily targets, and ORB will then manage the available tonnes to each draw point at any given time based on an ideal smooth progression toward that target over the month. Additional constraints are added to manage draw around this including:

- Maximum tonnes per draw point in a duration

- Minimum tonnes per draw point in a duration

- Permissible tonnes over smooth progression (i.e. permits over-bogging within an acceptable limit)

- Permissible tonnes under smooth progression (i.e. permits under-bogging within an acceptable limit)

- Maximum and minimum orderable tonnes (i.e. allowable size of a single dispatch or 'order')

- Planned unavailability (e.g. roadway repair restricting extraction drive access)

- Other, cave-specific constraints 
The draw point timed targets draw strategy can result in the removal of most of the shift start or daily planning processes. Tonnes targets are set by a draw control engineer for a given time period (e.g. weekly, fortnightly, monthly) and ORB dispatches LHDs in real-time to progress 'smoothly' toward these targets subject to continuous draw constraints. For example, rolling time windows are used such as 'maximum 300 tonnes in 12 hours' as opposed to shift-based constraints such as 'maximum 300 tonnes per shift'. Figure 1 shows an example of a draw point progression over a month.

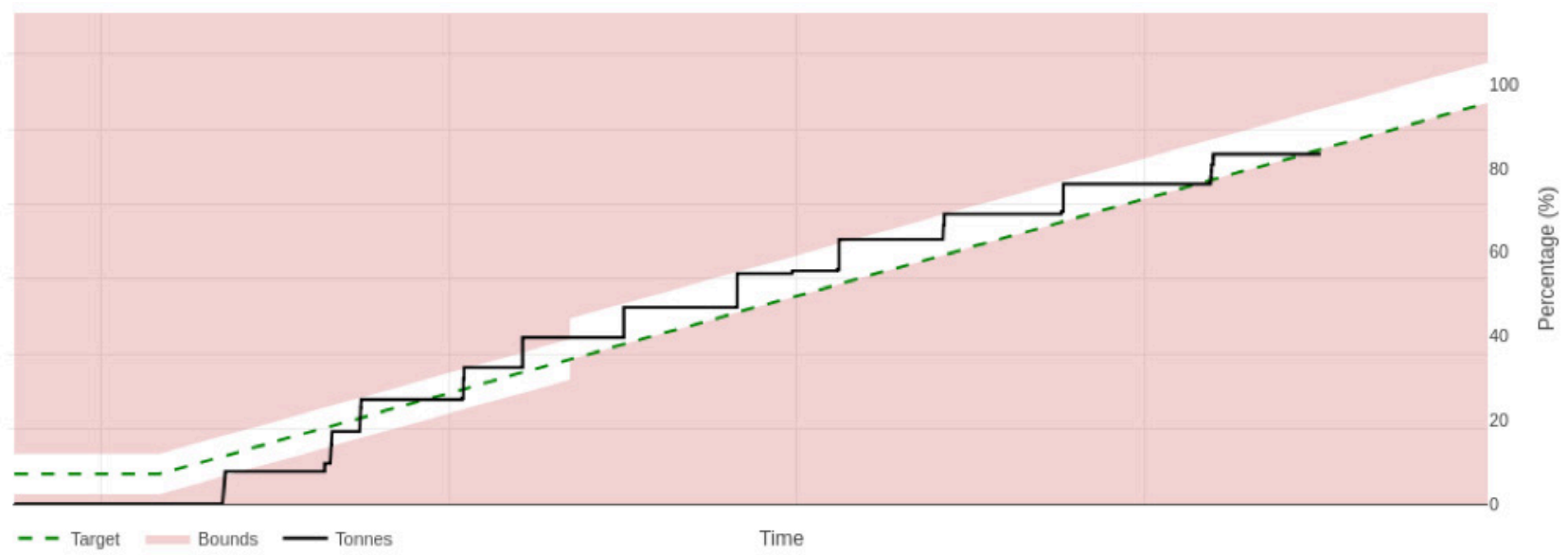

Figure 1 Example of Draw point Progression Chart, which displays how a draw point progresses towards a fixed tonnes target. Black line: The actual tonnes progression of the draw point to its target. Dashed green line: The theoretical smooth progression of the draw point to target under continuous bogging. This line flattens over periods of known downtime. White area: The allowable region for deviation from smooth compliance. Red area: Non-compliant region, if the actual tonnes progression is in this area then the draw point is non-compliant

Through integration with third-party data sources, ORB builds a digital twin of the real-time cave state. Data captured includes LHD tipping cycles (load/dump location and timing as well as tonnage volume), equipment status (location and activities of equipment over time), as well as draw point status (available draw points versus those requiring secondary break).

Through its optimisation engines, ORB then schedules and dispatches LHDs in order to achieve draw compliance, the measure of how close each draw point is to its current target within the month. With the optimisation engines, also comes automation of this process, which allows for a far more continuous and responsive planning process.

\subsection{Equipment extraction drive allocation}

ORB uses mathematical programming to optimise and automate the creation of the equipment timeline. This process efficiently evaluates a set of decision variables and constraints against a defined objective function, in order to find the optimal timeline.

The output is an allocation of equipment to extraction drives or stockpiles over time, with allocated tramming routes and dump locations (Figure 2). When drive blockages exist, for example from drive convergence, rehab, equipment break downs, or roadway repair, the set of tramming routes chosen can be very complex. A solution is only valid if for any given time period, the tramming routes selected can be validly operated by the available LHD fleet. From potentially billions of tramming route combinations, ORB selects the optimal set based on a multi-objective function that considers the number of available LHDs, LHD performance, draw compliance, and wetness blend (grade blend also possible). 


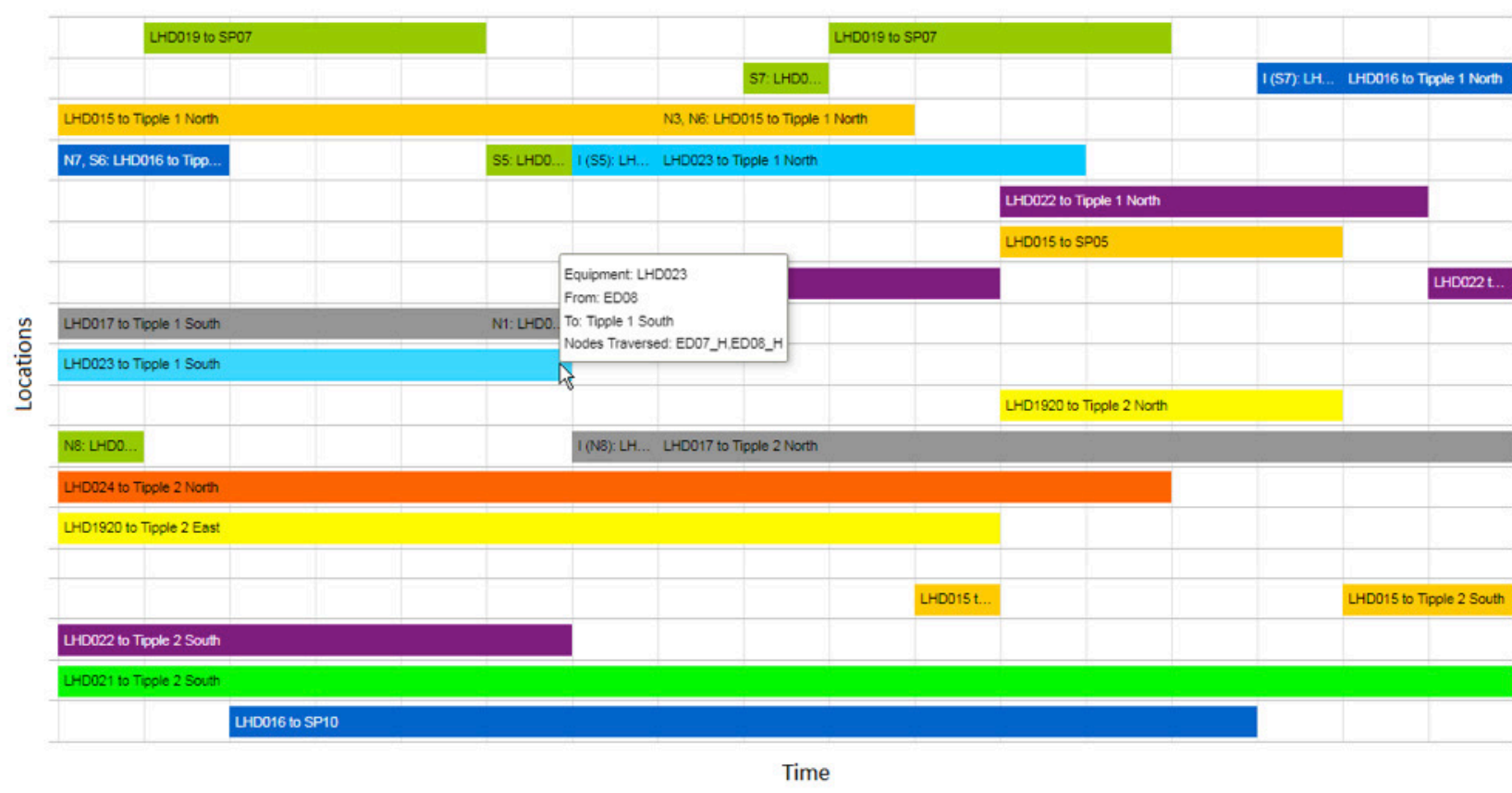

Figure 2 Output timeline of ORB Equipment Scheduler, displaying the allocation of equipment to extraction zones and tramming routes

\subsection{Real-time LHD draw point dispatch}

The ORB LHD Dispatch optimisation engine dispatches LHDs to draw points in real time using up-todate data to ensure the underground LHD fleet works together as an effective team. The optimisation simultaneously dispatches the current LHD (or LHDs) whose orders have ended and determines the best future dispatch decisions for the rest of the fleet that are nearing the end of their orders.

The objective of the optimisation process is a weighted combination of matching the capacity of the underground $\mathrm{MHS}$ and using the fleet as efficiently as possible, while ensuring smoothed progression towards targets and adhering to draw control constraints. Efficiency of fleet can be measured by the amount of time LHDs are doing productive work (loading, hauling, dumping) vs time spent waiting due to red lights or traffic. These relative weights are calibrated by the planner, dependent on the current priorities of the mine. The optimisation accounts for potential traffic and 'red light' stoppages caused by having too many LHDs near the tip point at once. Additionally, the optimisation can be configured to smooth ore grade and other attributes (e.g. wetness, hardness, fragmentation size) to within acceptable bounds to facilitate mine-to-mill initiatives.

\section{$2.4 \quad$ Short interval control}

With the deployment of optimisation-based decision-support tools come not only optimisation but also automation of these traditionally very manual processes. This means an operation can transition from traditional defence of a plan, to updating the plan based on actual events as they occur such as:

- Equipment returns from workshop

- Equipment breaks down

- Draw point hang up

- Rehabilitation or secondary break requirements

- Bogging faster / slower than expected

Throughout the day, as these events are captured through data integrations, a new plan can be optimised and published to operators through a process known as highly automated short interval control as defined by the Global Mining Guidelines Group (2019). Combined with the replacement of daily tonnes 
targets with those over a longer duration, this enables continuous planning and the removal of many artificial planning horizons.

\section{Case study: Rio Tinto's Argyle Diamonds}

\subsection{Argyle overview}

Argyle diamond mine is located in the remote East Kimberley region of Western Australia. It has been operating since 1983 initially as an alluvial operation then open pit, which ceased in 2013, before transitioning underground. Underground development was completed in June 2015, after more than 42 kilometres of tunnelling. The underground mine is the first block cave in Western Australia. The operation has produced more than $800 \mathrm{M}$ carats of rough diamonds making it one of the world's largest supplier of diamonds. 2020 will be the final year of production..

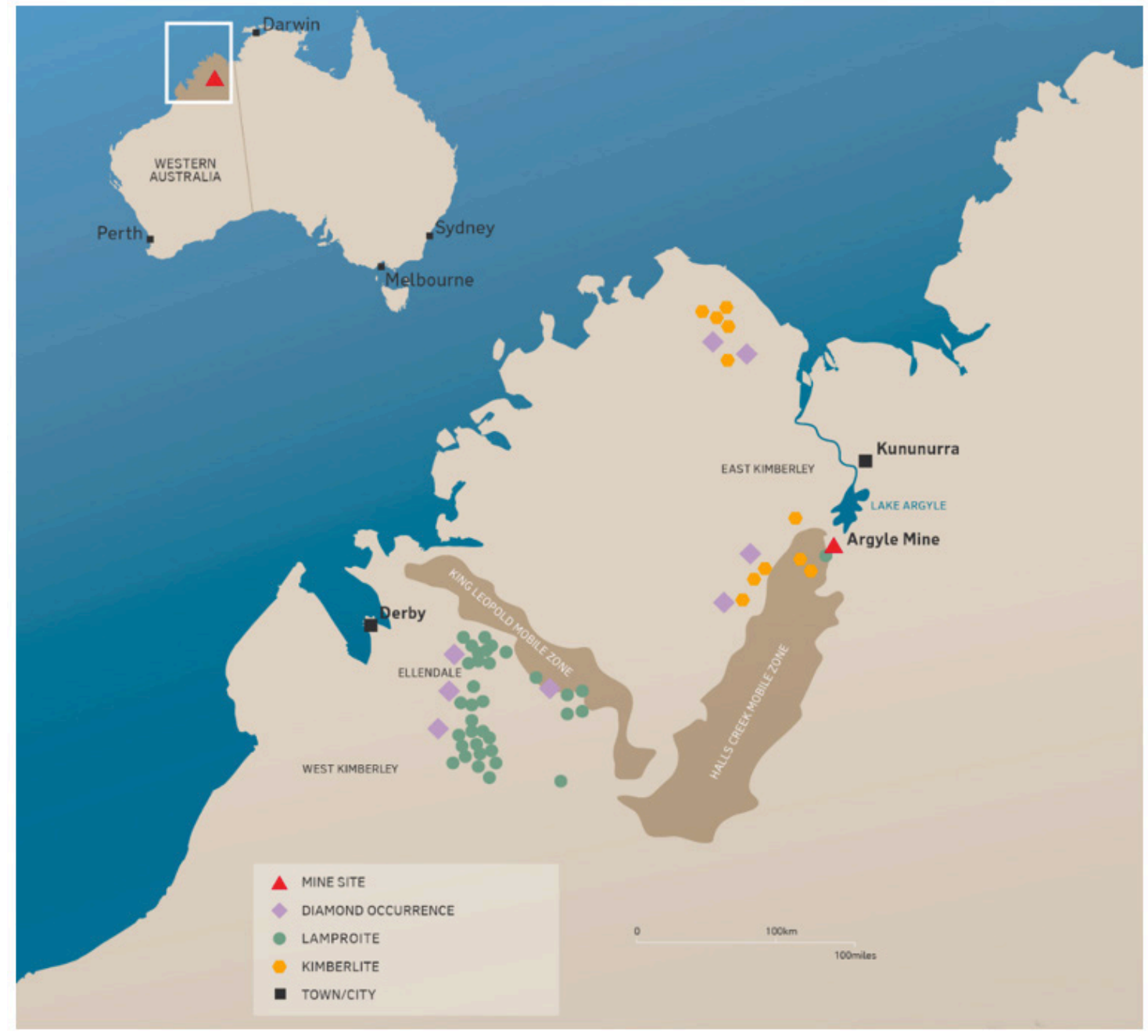

Figure 3 Location of Argyle diamond mine 


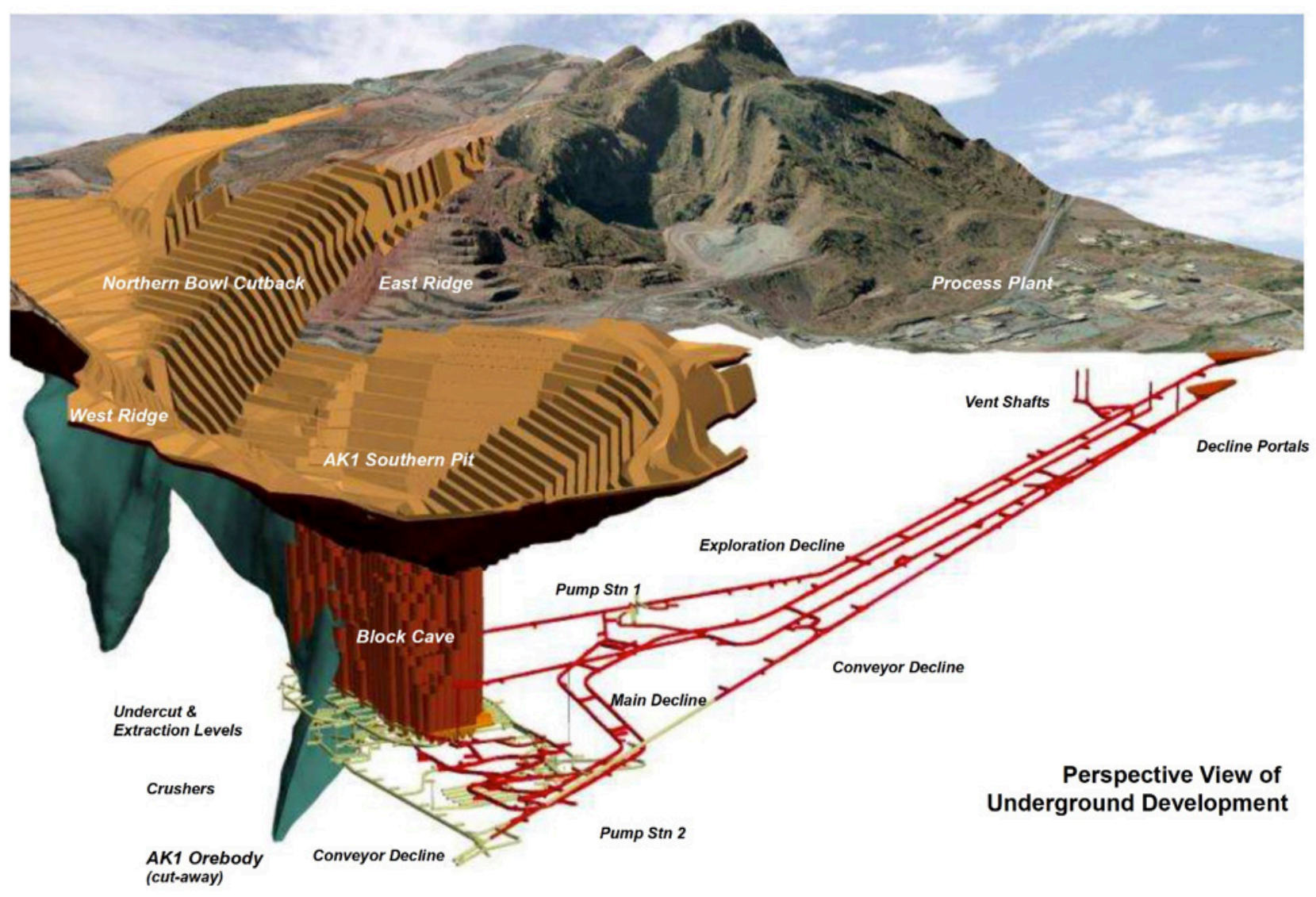

Figure 4 Layout of Argyle diamond mine and block cave

Argyle is a very complex operation with competing constraints including:

- 148 currently active draw points of originally 240 excavated

- Wet/dry blend ratio requirement (challenging during the 4-month long wet season)

- Differential crusher run rates

- Geotechnical considerations

- Overall cave health/draw strategy

- Blockages - due to roadway repairs, rehabilitation and secondary break

- 9 operating LHDs

- 3 different LHD sizes

- Zonal requirements limiting the number of LHDs in a particular area of the cave

- Drives and draw points which can only be accessed by a subset of the LHD fleet

Given the number of draw points and drives, ORB's Operational decisions require it to choose tramming route plans from up to 300,000 combinations for up to 9 LHDs. ORB needs to consider all the above constraints while choosing the set which optimises the productivity of the operation.

\subsection{Staged rollout}

A phased implementation of ORB LHD dispatch was selected to lessen the impact on pre-existing systems, work methodologies and instil confidence in the output for all stakeholders. 


\subsubsection{Phase 1: Operational}

Phase 1 consisted of the configuration of a tool to replace fixed daily timelines with dynamically optimised timelines that reflect current constraint statuses and actual tonnages achieved. The aim was to implement a system that could be updated throughout the shift as actual events occurred, however it was critical to progressively work toward this to minimise business process change.

Initially, the tool was rolled out and used to make a new plan at the start of the shift, using existing daily draw targets. This was then given to a production supervisor who could use this as a basis for the setup of their shift.

The project team worked closely with the production supervisors to obtain feedback as to the practically of the solution, and iteratively worked through issues as they arose. This process built trust in ORB's ability to make a 'good plan'.

An interface was then provided to the control room operators such that they could re-generate a plan in response to actual events throughout the shift. This allowed the team to stop defending a plan once it was out of date, and instead make a new one.

The first phase of the roll out used the pre-existing daily draw targets. This meant that ORB was able to optimise the timeline from any point in time, until the end of night shift. This was decided to minimise business process change, however created some difficulties near the end of night shift when there were only a few hours left to plan, and the available tonnes was very restricted. These issues were addressed in phase 2 with the introduction of monthly targets.

\subsubsection{Phase 2: Real-Time}

Phase 2 introduced real-time LHD dispatch to individual draw points, as well as the replacement of the daily draw targets with monthly targets. LHD dispatch was based on pre-defined parameters concerning wet/dry blending, geotechnical considerations, cave health, drive blockages and differing LHD sizes. Through integration with real-time production data, ORB was able to provide continuous and autonomous dispatch of LHDs for every order. These orders were then automatically pushed to LHD operators through integration with in-cab displays. These were also able to be used to capture draw points hang ups from the LHD operators as they happened.

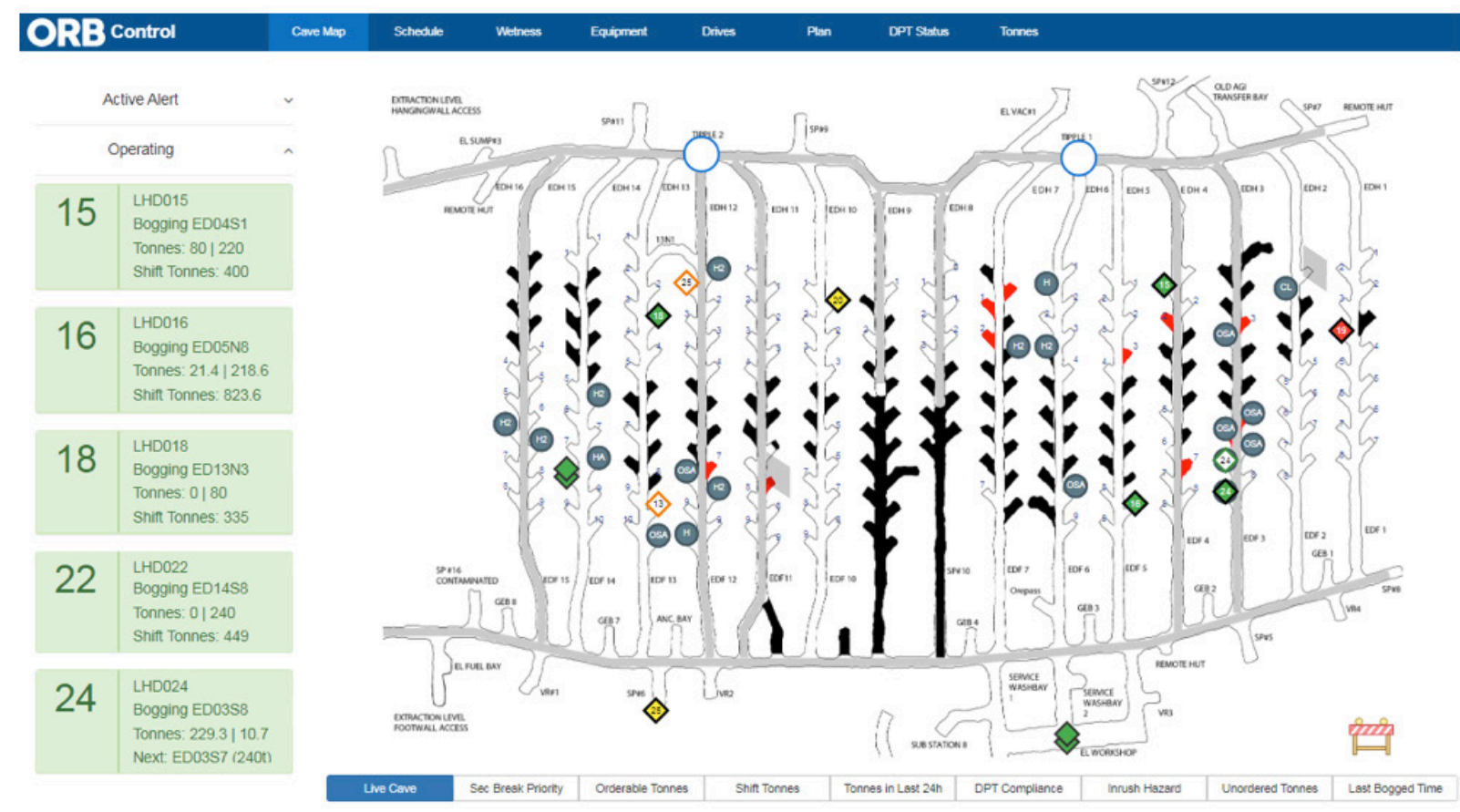

Figure 5 ORB dashboard displaying LHD dispatch decisions, task progress, and real-time cave state 
The transition to monthly targets allowed the execution of a more continuous draw strategy. Instead of optimising from the current time until the end of shift, the solve instead runs for a rolling period, for example the next 18 hours. Historically, a timeline was always created for 24 hours in order to match the duration of the daily draw targets. With a system that can efficiently make a new plan when the cave state changes, it is no longer required to have visibility of the entire next 24 hours' worth of decisions. Instead, the decision right now becomes the most important, as the plan can always be re-run throughout the shift should the actual performance of the cave differ from the plan. At times, this can mean a new plan is created multiple times per hour. It is important to note that when running a new plan, all current equipment activities are locked in to ensure only equipment that must move is re-allocated. This means that although a new plan is created, no unnecessary LHD movements are required.

In order to run the mathematical optimisations, ORB requires data feeds from several sources. Given the impact of the wet season at Argyle, it was particularly important that ORB was capturing data for both wetness blend requirements, and the real-time LHD tonnages. Using this, a custom module for ORB was developed to track, visualise, and alarm in real-time the wetness blend. Additionally, this information was incorporated into the optimisation such that ORB would dispatch to blend on wetness. The alarm then serves as a backup for when actual tonnages and LHD speeds differ to those predicted by the dispatch algorithm, and the wetness blend needs manual intervention.

\section{$4 \quad$ Results}

\subsection{Key learnings}

Several unique challenges arose in the deployment of ORB to Argyle, which resulted in the following key learnings:

- 'Buy-In' from all levels within an organisation is very important. Any change to existing business processes requires strong communication and justification amongst stakeholders.

- Managing attitude and culture were large parts of the roll-out process.

- Being responsive to feedback from end-users is critical to success.

- Large differences in computer literacy/proficiency required additional training.

- The complexities of the dispatch tasks that supervisors were previously expected to manually optimise were highlighted.

- Integration of new processes into legacy technology/data feeds sometimes requires workarounds.

- Real-time data capture sensors and associated infrastructure are no longer something to be tacked on as an afterthought, they are integral to running any operation and must be treated as such. (You wouldn't operate a LHD with a flat tyre so you shouldn't be operating with faulty telemetry).

- To keep pace mining must embrace a more data-centric model that integrates 'applied mathematics' into complex decision-making.

\subsection{Benefits}

The primary benefits have included:

- Significantly Improved cave compliance with a $15 \%+$ increase in combined draw point monthly weighted average cave compliance. This has been crucial at this stage of the operation to help control grade and cave health.

- Improved tonnage over shift-change (3hr window) $\approx 7 \%$ increase directly attributable to ORB when normalised for increases in bucket size and LHD operational hours. This is due to the transition from daily to monthly targets, and the corresponding removal of the planning reset at the start of each day. 
- Improved overall tonnage resulted in 2019 being the most productive year ever for the underground operation (5.9Mt), with an increase of 18.5\% compared to 2018 . Various factors contributed to this achievement with a large proportion of this additional tonnage the result of increased mining productivity which has been underpinned by ORB.

Secondary benefits of the system:

- Ability to react quickly and consistently when issues arise.

- Consistent decision making across crews/supervisors through decision automation.

- Increased supervisor focus on personnel management as a result of less time spent on scheduling decisions.

- Engineer workload can be refocussed on longer term planning horizon through the removal of tedious daily manual processes.

- LHDs no longer run out of tonnes at the end of night shift, due to the continuous release of tonnes over a longer time period.

- There are now different mechanisms for constraining draw tonnes, which more closely align with caving and operational requirements than daily targets. This allows engineers to be deliberately constraining where required and give more draw flexibility where possible. Targets no longer need to be set daily, and instead monitored and tweaked over time as compliance is monitored. This has in general resulted in increased tonnage availability across the cave, which leads to LHDs being able to bog in the same drive for longer and thus have less downtime transitioning between drive. Furthermore, this increased flexibility in draw point tonnage has resulted in improved cave compliance.

\section{Conclusions}

In conclusion, this paper aimed to describe the configuration and implementation of ORB, the first known optimisation-based, autonomous, real-time dispatch tool custom built for underground block cave mining operations. Through continuous and autonomous co-ordination of the optimal allocation and dispatch of LHDs, it is able to achieve a range of objectives while adhering to draw compliance and geotechnical constraints. As presented through a case study at Rio Tinto's Argyle Diamonds, it has been shown that ORB has the capacity to improve both tonnes to surface and draw compliance.

\section{Acknowledgement}

This paper summarises the extensive work of a small team from Rio Tinto and Polymathian, and the authors would like to acknowledge their efforts during the project and in preparing this paper.

\section{References}

Global Mining Guidelines Group 2019, 'Guideline for implementing short interval control in underground mining operations', 1st ed. Available at https://gmggroup.org/wp-content/uploads/2019/06/20181015_SIC-GMG-UM-v01-r01.pdf Accessed 1/9/2019.

Hustrulid, WA 2001, 'Underground Mining Methods: Engineering Fundamentals and International Case Studies', Society for Mining, Metallurgy, and Exploration (SME), Littleton.

Khodayari, F \& Pourrahimian, Y 2015, 'Mathematical programming applications in block-caving scheduling: A review of models and algorithms', International Journal of Mining and Mineral Engineering, vol. 6, pp. 234-257.

Paravarzar, S, Pourrahimian, Y \& Askari Nasab, H 2018, 'Short-Term Underground Mine Planning: A Review', Mining Optimization Laboratory (MOL), paper 309, pp. 305-334. 